Cahiers québécois de démographie

\title{
Vieillissement et incapacités au Québec : perspectives
} régionales 1991-2006

\section{AGING AND DISABILITY IN QUEBEC: REGIONAL PROJECTIONS 1991-2006 ENVEJECIMIENTO E INCAPACIDAD EN QUEBEC : PERSPECTIVAS REGIONALES 1991-2006}

\section{Anne Bernard, Yves Bussière et Jean-Pierre Thouez}

Volume 26, numéro 1, printemps 1997

La santé (suite)

URI : https://id.erudit.org/iderudit/010226ar

DOI : https://doi.org/10.7202/010226ar

Aller au sommaire du numéro

Éditeur(s)

Association des démographes du Québec

ISSN

0380-1721 (imprimé)

1705-1495 (numérique)

Découvrir la revue

Citer cet article

Bernard, A., Bussière, Y. \& Thouez, J.-P. (1997). Vieillissement et incapacités au Québec : perspectives régionales 1991-2006. Cahiers québécois de démographie, 26(1), 91-107. https://doi.org/10.7202/010226ar
Résumé de l'article

Le vieillissement rapide de la population québécoise au cours des prochaines décennies entraînera des disparités régionales importantes. À partir de projections démographiques régionales du Bureau de la statistique du Québec (BSQ) sur la période 1991-2006 et des données de l'Enquête sur la santé et les limitations d'activités de Statistique Canada (ESLA), nous présentons une analyse de l'évolution des clientèles à mobilité réduite. L'analyse démontre non seulement l'importance des disparités régionales mais également leur impact sur l'évolution des incapacités restreignant la mobilité. 
Cahiers québécois de démographie

Vol. 26, no 1, printemps 1997, p. 91-107.

\title{
Vieillissement et incapacités au Québec: perspectives régionales 1991-2006
}

\author{
Anne BERNARD, Yves BUSSIÈRE et Jean-Pierre THOUEZ *
}

Le vieillissement rapide de la population québécoise au cours des prochaines décennies soulève des questions sur l'ampleur des clientèles âgées en perte d'autonomie à satisfaire en termes de logement, de services sociaux, de santé et de transport. L'objectif de cet article est d'étudier le vieillissement démographique observé au Québec en relation avec la notion de mobilité des individus. L'accélération attendue du phénomène de vieillissement de la population, sa féminisation, l'augmentation avec l'âge des taux de prévalence des incapacités qui réduisent la mobilité des individus, l'existence de générations moins mobiles (notamment de femmes âgées sans permis de conduire et vivant dans des banlieues mal desservies par les transports collectifs), les divers impacts de la perte d'autonomie sur les personnes âgées et sur leur entourage, ses effets sur la demande de services de santé ou de transport, et enfin l'impact des modifications de cette demande sur la forme urbaine et sur l'aménagement des réseaux de services sont autant de raisons qui légitiment l'intérêt accordé à la relation entre vieillissement et incapacité.

Cet article reprend en partie les résultats d'une recherche plus large réalisée récemment pour le ministère québécois des Transports auprès de la population non institutionnalisée du Québec, et portant sur la demande de transport des personnes à mobilité réduite (Bussière et al., 1996). Nous nous attarderons ici sur la mobilité définie à partir d'une série de critères tirés de

Anne Bernard et Yves Bussière : INRS-Urbanisation, Institut national de la recherche scientifique, Université du Québec; Jean-Pierre Thouez : Département de Géographie, Université de Montréal. Nous remercions le ministère des Transports du Québec et le Fonds pour la formation de chercheurs et l'aide à la recherche du Québec de leur appui. 
l'Enquête sur la santé et les limitations d'activités (ESLA), et nous tenterons d'évaluer l'évolution possible des incapacités à l'horizon 2006 à partir de l'année 1991.

\section{DONNÉES ET MÉTHODE}

\section{Données}

Les données démographiques proviennent du recensement de 1991 (Statistique Canada) et des estimations de la population en 2006 pour le Québec et ses seize régions administratives réalisées par le Bureau de la statistique du Québec (BSQ) ${ }^{1}$. Des cinq scénarios établis par le BSQ, le scénario de référence a été retenu : espérance de vie équivalant à un gain d'environ 3,3 ans entre 1991 et 2011 ; indice synthétique de fécondité de 1,6 atteint en 1993; solde total des migrations de 25000 , atteint en 1994 (voir aussi Mathews, 1996).

Les données sur les incapacités proviennent de l'Enquète sur la santé et les limitations d'activités (ESLA) de 1986-1987 réalisée par Statistique Canada et concernent les personnes âgées de 15 ans et plus vivant dans un ménage privé. Bien qu'il existe une enquête ESLA plus récente (1991), nous avons utilisé celle de 1986-1987, qui a porté sur un échantillon de plus grande taille que celle de 1991 (5390 plutôt que 1927 répondants vivant à domicile au Québec). De plus, l'enquête de 1991 ne ventile pas les données sur les personnes âgées de 65 ans ou plus vivant dans les régions métropolitaines, et ne permet pas une analyse désagrégée par région administrative.

\section{Méthode}

Chez un individu, la mobilité réduite peut provenir d'une incapacité ou d'une combinaison d'incapacités plus ou moins grave(s). Une personne à mobilité réduite est toute personne dont la capacité de déplacement est réduite par une ou plusieurs déficience(s) intellectuelle(s), physique(s) ou sensorielle(s), même lorsque cette ou ces déficience(s), permanente(s) ou non, résulte(nt) du processus normal de vieillissement et (ou) de problèmes de santé, temporaires ou chroniques (voir entre autres

1 Bas-Saint-Laurent, Saguenay-Lac-Saint-Jean, Québec, Mauricie-BoisFrancs, Estrie, Montréal, Outaouais, Abitibi-Témiscamingue, Côte-Nord, Nord-du-Québec, Gaspésie-Îles-de-la-Madeleine, Chaudière-Appalaches, Laval, Lanaudière, Laurentides et Montérégie. 
Guyon et Levasseur, 1991; Bussière et al., 1996). Mais la perte d'autonomie est d'autant plus difficile à évaluer que la mesure de l'incapacité varie selon les enquêtes (Colvez et al., 1990).

Notre définition de l'incapacité est celle retenue par le ministère des Transports du Québec (MTQ) pour l'admissibilité au service de transport adapté (Bussière et al., 1996). Ainsi, les personnes à mobilité réduite vivant dans les ménages ont été regroupées en quatre catégories : incapacité locomotrice ambulatoire (la personne peut se déplacer en marchant), incapacité locomotrice non ambulatoire (la personne se déplace en fauteuil roulant), incapacité intellectuelle et incapacité visuelle (tableau 1).

Pour tenir compte des chevauchements et ne retenir qu'une seule incapacité lorsque les incapacités sont combinées chez un même individu, nous avons établi un ordre de priorité : priorité est accordée à l'incapacitê locomotrice non ambulatoire quand elle est combinée à une autre incapacité, et aux incapacités intellectuelle et visuelle lorsqu'elles sont combinées à l'incapacité locomotrice ambulatoire; seule l'incapacité intellectuelle est retenue lorsqu'elle est combinée à l'incapacité visuelle.

TABLEAU 1 - Définition des incapacités

\begin{tabular}{ll}
\hline \multicolumn{1}{c}{ CATÉGORIES } & \multicolumn{1}{c}{ CRITÈres } \\
\hline $\begin{array}{l}\text { 1- Incapacité locomotrice } \\
\text { ambulatoire }\end{array}$ & $\begin{array}{l}\text { Difficulté à marcher } 350 \mathrm{~m} \text { sans repos } \\
\text { Difficultè à monter et à descendre un escalier } \\
\text { Difficulté à transporter un objet de } 4,5 \mathrm{~kg} \text { sur } \\
10 \mathrm{~m}\end{array}$ \\
$\begin{array}{l}\text { Incapacité locomotrice } \\
\text { non ambulatoire } \\
\text { (fauteuil roulant) }\end{array}$ & $\begin{array}{l}\text { Difficulté à se tenir debout plus de } 20 \mathrm{mn} \\
\text { Difficulté à se pencher et à ramasser un objet } \\
\text { Difficulté à se servir de ses doigts } \\
\end{array}$ \\
& Difficulté à ètendre les bras \\
\hline
\end{tabular}

3- Incapacité intellectuelle Difficulté à parler et à ètre compris

Problèmes de mémoire et (ou) d'apprentissage

4- Incapacité visuelle

Reconnu aveugle (par un médecin)

Note : La personne qui répond à l'un des critères énumérés et dont l'affection a duré ou devrait durer au moins six mois est considérée dans l'ESLA comme une personne ayant une incapacité. Par contre, n'est pas classée comme ayant une incapacité la personne qui utilise une aide technique lui permettant de surmonter ses limitations. 
Nous avons calculé les taux de prévalence de ces incapacités dans la population en rapportant les fréquences (tirées de l'ESLA) aux données de population du recensement de 1986. Ces taux de prévalence ont ensuite été appliqués aux structures par âge et sexe des populations régionales en 1991 et 2006 . À cet égard, l'information provenant de l'ESLA 1986 étant déficiente en ce qui concerne la population avec incapacité dans les régions, nous avons construit une grille d'approximation : à la population de chacune des régions, nous avons appliqué, selon le cas, les taux de prévalence des incapacités provenant de l'ESLA 1986 au niveau soit de la région métropolitaine de Montréal (Montréal, Laval), soit des autres régions urbaines du Québec (Québec, Outaouais, Montérégie), soit des onze autres régions du Québec (Bas-Saint-Laurent, Saguenay-Lac-SaintJean, Mauricie-Bois-Francs, Estrie, Abitibi-Témiscamingue, Côte-Nord, Nord-du-Québec, Gaspésie-Îles-de-la-Madeleine, Chaudière-Appalaches, Lanaudière et Laurentides). Les calculs ont été faits selon l'âge, le sexe et la région administrative.

\section{DÉMOGRAPHIE ET INCAPACITÉ}

\section{Les taux de prévalence des incapacités en 1986}

Selon l'ESLA 1986, les taux de prévalence pour cent mille habitants sont de 6558 pour l'incapacité locomotrice ambulatoire, de 2730 pour l'incapacité intellectuelle, de 391 pour l'incapacité locomotrice non ambulatoire (fauteuil roulant) et de 93 pour l'incapacité visuelle.

\section{L'incapacité selon l'âge, la gravité et le sexe en 1986}

Les taux de prévalence des incapacités selon l'ESLA 1986 augmentent sensiblement avec l'âge de l'individu, notamment lorsqu'on passe de la tranche d'âge des 65-74 ans à celle des 75 ans ou plus (figure 1) (voir aussi Thouez et al., 1993).

Le profil des incapacités selon la gravité (indice créé par Statistique Canada à partir des données de l'ESLA par la pondération des diverses incapacités) révèle que c'est pour l'incapacité modérée que les taux de prévalence sont les plus élevés dans la population (tableau 2). Par ailleurs, le taux de prévalence des incapacités semble plus élevé pour les femmes que pour les hommes chez les 75 ans ou plus (figure 2) (voir aussi Saucier et Lafontaine, 1991; Saucier, 1992; Thouez et al., 1993). 


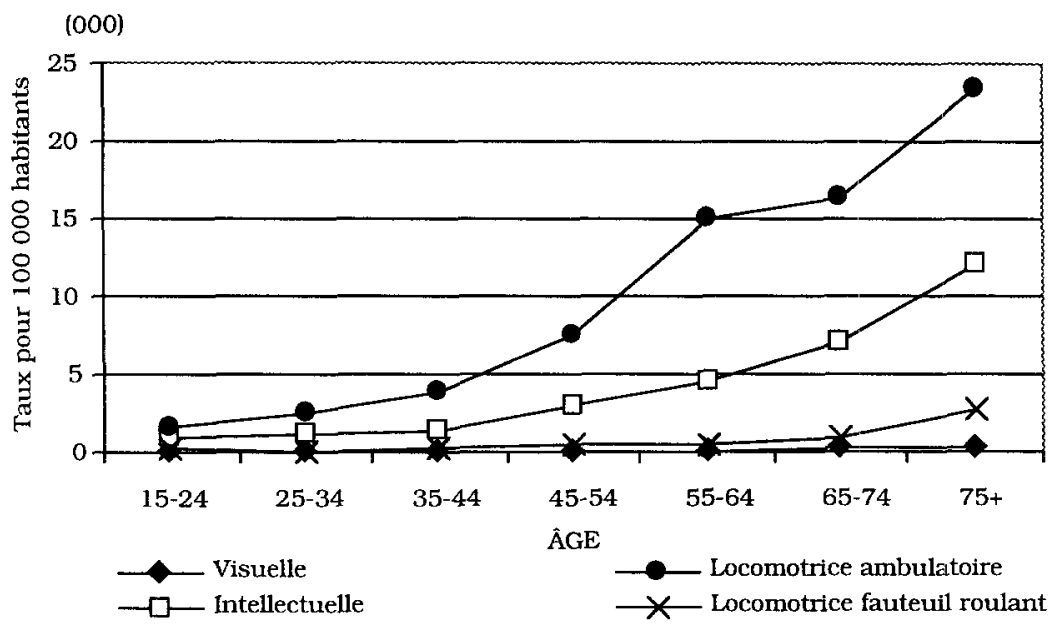

Source : ESLA 1986; traitements INRS.

FIGURE 1 - Taux de prévalence des incapacités

par catégorie d'âge au Québec, 1986

\section{L'incapacité au guébec en 1991}

L'application des taux de prévalence par âge et sexe tirés de l'ESLA 1986 aux données de population du recensement de 1991 permet de dégager certaines caractéristiques globales de la population avec incapacité : au Québec, en 1991, on observe, parmi les 589306 personnes âgées de 15 ans ou plus vivant dans les ménages et ayant une incapacité, une proportion majoritaire de personnes atteintes d'une incapacité locomotrice ambulatoire $(67,6$ pour cent), une forte proportion d'indi-

TABLEAU 2 - Taux de prévalence des incapacités réduisant la mobilité, par catégorie et gravité, Québec, 1986 (pour 100000 habitants)

\begin{tabular}{lrrrrr}
\hline & \multicolumn{4}{c}{ Gravité } \\
Incapacité & Légère & Modérée & Grave & Total \\
\hline Locomotrice ambulatoire & 2278 & 3 & 041 & 1239 & 6558 \\
Locomotrice fauteuil roulant & 17 & 47 & 327 & 391 \\
Intellectuelle $_{\text {Visuelle a }}$ & 706 & 1 & 066 & 848 & 2620 \\
Total & 2 & 35 & 29 & 66 \\
\hline
\end{tabular}

Source : ESLA 1986; traitements INRS.

a. Données à interpréter avec prudence étant donné la faible taille de l'échantillon. 


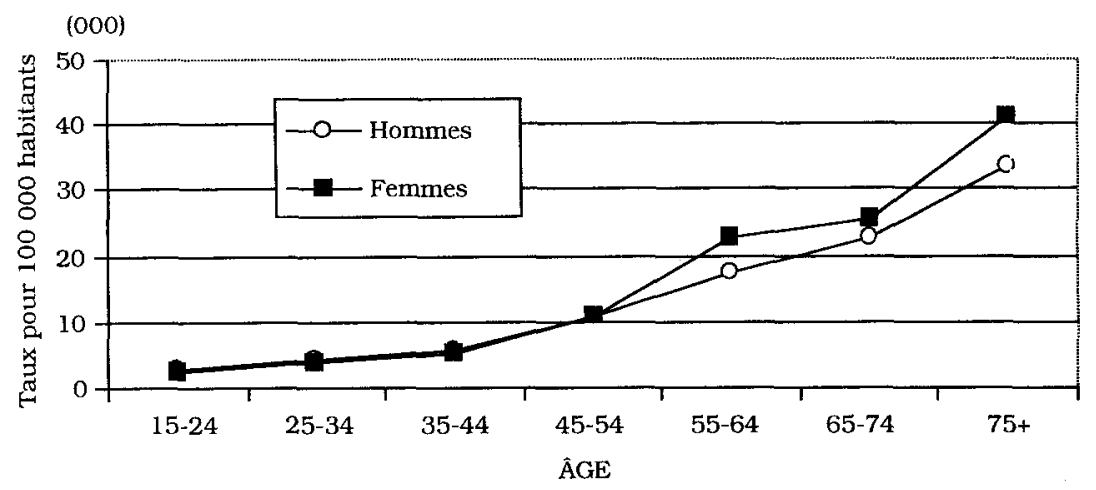

Source : ESLA 1986; traitements INRS.

FIGURE 2 - Taux de prévalence des incapacités selon l'áge et le sexe, Québec, 1986

vidus touchés par une incapacité intellectuelle ( 27,5 pour cent), une surreprésentation des personnes âgées de 55 à 64 ans dans la population touchée par une incapacité locomotrice ambulatoire, une augmentation avec l'âge du nombre de personnes ayant une incapacité (notamment pour la tranche d'âge des 75 ans ou plus), et une concentration dans les régions fortement urbanisées des personnes à mobilité rẻduite (tableaux 3 et 4).

En 1991, on estime à 232900 le nombre de personnes âgées de 65 ans ou plus qui sont affectées par une incapacité (soit près de 30 pour cent des personnes de cette tranche d'âge). Pour 64 pour cent de ces personnes, il s'agit d'une incapacité locomotrice ambulatoire (et pour 30 pour cent d'une incapacité intellectuelle) (tableau 3). En 1991, le tiers (33 pour cent) des personnes âgées de 65 ans ou plus ayant une incapacité habi-

TABLEAU 3 - Population âgée de 15 ans ou plus ou âgée de 65 ans ou plus avec une incapacité, selon la catégorie d'incapacité, Québec, 1991

\begin{tabular}{|c|c|c|c|c|}
\hline \multirow[b]{2}{*}{ Incapacité } & \multicolumn{2}{|c|}{ Pop. 15 ans +} & \multicolumn{2}{|c|}{ Pop. 65 ans +} \\
\hline & $\mathrm{N}$ & $\%$ & $\mathrm{~N}$ & $\%$ \\
\hline Locomotrice ambulatoire & 398735 & 67,6 & 148899 & 63,9 \\
\hline Locomotrice fauteuil roulant & 24105 & 4,1 & 12357 & 5,3 \\
\hline Intellectuelle & 162390 & 27,6 & 70012 & 30,1 \\
\hline Visuelle & 4076 & 0,7 & 1632 & 0,7 \\
\hline Total & 589306 & 100,0 & 232900 & 100,0 \\
\hline
\end{tabular}

Source : BSQ, ESLA 1986; traitements INRS. 
TABLEAU 4 - Population âgée de 15 ans ou plus ou âgée de 65 ans ou plus avec une incapacité, selon la région, Québec, 1991

\begin{tabular}{|c|c|c|c|c|}
\hline \multirow{3}{*}{$\begin{array}{l}\text { Région } \\
\text { administrative }\end{array}$} & \multicolumn{4}{|c|}{ POPULATION AVEC INCAPACITE } \\
\hline & \multicolumn{2}{|c|}{ Pop. 15 ans +} & \multicolumn{2}{|c|}{ Pop. 65 ans +} \\
\hline & $\mathrm{N}$ & $\%$ & $\mathrm{~N}$ & $\%$ \\
\hline Bas-Saint-Laurent & 17787 & 3,0 & 7702 & 3,3 \\
\hline Saguenay-Lac-Saint-Jean & 21418 & 3,6 & 7232 & 3,1 \\
\hline Québec & 59340 & 10,1 & 22742 & 9,8 \\
\hline Mauricie-Bois-Francs & 41026 & 7,0 & 17206 & 7,4 \\
\hline Estrie & 23042 & 3,9 & 9801 & 4,2 \\
\hline Montréal & 163765 & 27,8 & 77213 & 33,2 \\
\hline Outaouais & 23464 & 4,0 & 7686 & 3,3 \\
\hline Abitibi-Témiscamingue & 10963 & 1,9 & 3757 & 1,6 \\
\hline Cöte-Nord & 6895 & 1,2 & 1760 & 0,8 \\
\hline Nord-du-Québec & 1766 & 0,3 & 297 & 0,1 \\
\hline Gaspésie-Îles-de-la-Madeleine & 8948 & 1,5 & 3645 & 1,6 \\
\hline Chaudière-Appalaches & 29565 & 5,0 & 11745 & 5,0 \\
\hline Laval & 23762 & 4,0 & 8168 & 3,5 \\
\hline Lanaudière & 24577 & 4,2 & 8046 & 3,5 \\
\hline Laurentides & 29331 & 5,0 & 10246 & 4,4 \\
\hline Montérégie & 103656 & 17,6 & 35654 & 15,3 \\
\hline Ensemble du Québec & 589305 & 100,0 & 232900 & 100,0 \\
\hline
\end{tabular}

Source : d'après Bussière et al., 1996.

taient dans la région métropolitaine de Montréal, et près d'un autre tiers (32 pour cent) habitaient dans une des trois régions administratives suivantes: Montérégie, Québec et MauricieBois-Francs (tableau 4).

\section{ÉVOLUTION DÉMOGRAPHIGUE PROJETÉE AU GUÉBEC, 1991-2006}

\section{Évolution démographique au Québec, 1991-2006}

Selon le scénario de référence du BSQ, la population du Québec devrait connaitre une croissance de 17 pour cent durant la période 1991-2006. La population âgée de 15 ans ou plus pourrait croitre de 11,5 pour cent au cours de la même période (croissance annuelle de 0,7 pour cent : tableau 5). Le nombre de femmes en âge de procréer (âgées de 15 à 39 ans) devrait subir un déclin lent mais régulier, tandis que le nombre de personnes âgées devrait augmenter sans cesse, d'où une courbe des naissances légèrement descendante et une courbe des décès ascen- 
TABLEAU 5 - Évolution de la population totale par groupe d'âge et sexe, Québec, 1991-2006

\begin{tabular}{|c|c|c|c|c|}
\hline$\hat{A} G E$ & 1991 & 2006 & $\begin{array}{l}\text { Croissance } \\
\text { globale }(\%)\end{array}$ & $\begin{array}{l}\text { Croissance } \\
\text { annuelle }(\%)\end{array}$ \\
\hline \multicolumn{5}{|c|}{ HoMMES } \\
\hline $0-14$ & $\begin{array}{lll}718 & 072\end{array}$ & 686340 & $-4,4$ & $-0,3$ \\
\hline $15-24$ & 494013 & 504777 & 2,2 & 0,1 \\
\hline $25-34$ & 660665 & 540352 & $-18,2$ & $-1,3$ \\
\hline $35-44$ & 583624 & 607239 & 4,0 & 0,3 \\
\hline $45-54$ & 407706 & $636 \quad 631$ & 56,1 & 3,0 \\
\hline $55-64$ & 309591 & 475894 & 53,7 & 2,9 \\
\hline $65-74$ & 209318 & 271067 & 29,5 & 1,7 \\
\hline $75+$ & 106805 & 180450 & 69,0 & 3,6 \\
\hline Total & 3489794 & 3902750 & 11,8 & 0,7 \\
\hline \multicolumn{5}{|c|}{ FEMMES } \\
\hline $0-14$ & 684753 & 646463 & $-5,6$ & $-0,4$ \\
\hline $15-24$ & 475387 & 480772 & 1,1 & 0,1 \\
\hline $25-34$ & $640 \quad 417$ & 511738 & $-20,1$ & $-1,5$ \\
\hline $35-44$ & 578914 & 586342 & 1,3 & 0,1 \\
\hline $45-54$ & 411715 & 637522 & 54,8 & 3,0 \\
\hline $55-64$ & 335204 & 494088 & 47,4 & 2,6 \\
\hline $65-74$ & 269576 & 316026 & 17,2 & 1,1 \\
\hline $75+$ & 195473 & 322761 & 65,1 & 3,4 \\
\hline Total & 3591439 & 3995712 & 11,3 & 0,7 \\
\hline \multicolumn{5}{|c|}{ TOTAL } \\
\hline $0-14$ & 1402825 & 1332803 & $-5,0$ & $-0,3$ \\
\hline $15-24$ & 969400 & 985549 & 1,7 & 0,1 \\
\hline $25-34$ & 1301082 & 1052090 & $-19,1$ & $-1,4$ \\
\hline $35-44$ & 1162538 & 1193581 & 2,7 & 0,2 \\
\hline $45-54$ & 819421 & 1274153 & 55,5 & 3,0 \\
\hline $55-64$ & 644795 & 969982 & 50,4 & 2,8 \\
\hline $65-74$ & 478894 & $\begin{array}{ll}587 & 093\end{array}$ & 22,6 & 1,4 \\
\hline $75+$ & 302278 & 503211 & 66,5 & 3,5 \\
\hline Total & 7081233 & 7898462 & 11,5 & 0,7 \\
\hline
\end{tabular}

Source : BSQ; traitements INRS.

dante (Mathews, 1988; Mathews, 1996; Bussière et al., 1996). Le poids des personnes âgées dans l'ensemble de la population âgẻe de 15 ans ou plus devrait passer, selon les projections, de 13,7 à 15,9 pour cent durant la période 1991-2006 (tableau 6).

Le vieillissement de la population se distingue de l'augmentation du nombre absolu de personnes âgées; or c'est cette der- 
TABLEAU 6 - Évolution de la population par région, Québec, 19912006

\begin{tabular}{|c|c|c|c|c|c|c|}
\hline \multirow[b]{2}{*}{$\begin{array}{l}\text { Région } \\
\text { administrative }\end{array}$} & \multicolumn{3}{|c|}{ Population 15 ans ou +} & \multicolumn{3}{|c|}{ Population 65 ans ou +} \\
\hline & 1991 & 2006 & $\begin{array}{c}\text { Croiss. } \\
\text { ann. } \\
(\%)\end{array}$ & 1991 & 2006 & $\begin{array}{c}\text { Croiss. } \\
\text { ann. } \\
(\%)\end{array}$ \\
\hline Bas-Saint-Laurent & 165677 & 163180 & $-0,1$ & 27070 & 32791 & 1,3 \\
\hline Sagu.-Lac-St-Jean & 224812 & 230926 & 0,2 & 25824 & 37456 & 2,5 \\
\hline Québec & 520746 & 568836 & 0,6 & 71875 & 101028 & 2,3 \\
\hline Mauricie-Bois-Fr. & 379880 & 422133 & 0,7 & 59969 & 79954 & 1,9 \\
\hline Estrie & 217699 & 244081 & 0,8 & 34101 & 43179 & 1,6 \\
\hline Montréal & 1535577 & 1618060 & 0,3 & 255418 & 309636 & 1,3 \\
\hline Outaouais & 229945 & 298695 & 1,8 & 24523 & 40236 & 3,4 \\
\hline Abitibi-Témiscam. & 119143 & 131381 & 0,7 & 13500 & 17377 & 1,7 \\
\hline Côte-Nord & 81877 & 82957 & 0,1 & 6375 & 11640 & 4,1 \\
\hline Nord-du-Québec & 25418 & 28250 & 0,7 & 1101 & 2135 & 4,5 \\
\hline Gasp--Îles-de-la-M. & 86403 & 81176 & $-0,4$ & 12857 & 15685 & 1,3 \\
\hline Chauđière-Appal. & 292479 & 327928 & 0,8 & 41253 & 54623 & 1,9 \\
\hline Laval & 258671 & 317907 & 1,4 & 28782 & 51180 & 3,9 \\
\hline Lanaudière & 263999 & 370421 & 2,3 & 28717 & 48929 & 3,6 \\
\hline Laurentides & 306329 & 440276 & 2,4 & 36522 & 60035 & 3.4 \\
\hline Montérégie & 969753 & 1240022 & 1,7 & 113285 & 184420 & 3,3 \\
\hline Ens. du Québec & 5678408 & 6565659 & 1,0 & $781 \quad 172$ & 1090304 & 2,2 \\
\hline
\end{tabular}

Source : BSQ, ESLA 1986; traitements INRS.

nière variable qui est importante pour l'analyse de l'évolution des services, notamment des transports publics. Par ailleurs, le groupe des personnes âgées de 65 ans ou plus constitue un ensemble très hétérogène où deux sous-groupes sont à distinguer, les 65-74 ans et les 75 ans ou plus, dont le nombre d'individus devrait croître d'environ 66 pour cent durant la période 1991-2006 (tableau 5). Par ailleurs, le rythme (qui sera particulièrement rapide au Guébec) auquel se réalise le vieillissement importe tout autant que le seuil atteint; aussi le Québec pourrait-il devenir une sorte de laboratoire social à cet égard (Mathews, 1988; Mathews, 1996; Bussière et al., 1996).

\section{Vieillissement selon les régions, 1991-2006}

Les perspectives régionales du BSQ révèlent que les grandes tendances mentionnées ci-dessus vont connaître des variantes régionales importantes. Les différences régionales relatives à la fécondité et à l'espérance de vie, qui se sont rétrécies avec le temps, sont maintenues constantes au cours de la période de 
projection. C'est au niveau des relations migratoires que se joue la distinction entre les régions et que s'explique leur plus ou moins grand dynanisme démographique (Bussière et al., 1996; Mathews, 1996). En fait, seulement quelques régions périphériques devraient voir leur population totale décliner durant la période 1991-2006, soit la Côte-Nord, la Gaspésie-Îles-de-laMadeleine, le Bas-Saint-Laurent et le Saguenay-Lac-SaintJean. Les autres régions devraient connaître une période de croissance assez longue, et généralement lente, avant de subir une baisse de leur population. Deux régions devraient enregistrer un léger déclin de leur population âgée de 15 ans ou plus au cours de la période 1991-2006, soit le Bas-Saint-Laurent et la Gaspésie-Îles-de-la-Madeleine (tableau 6).

Il faut cependant souligner qu'au niveau régional, une forte proportion de personnes âgées dans la population totale en 2006 correspond à une faible progression du nombre absolu de personnes âgées; de même, à une faible proportion de personnes âgées correspond généralement une forte progression de leur nombre. Ainsi, durant la période 1991-2006, le nombre de personnes âgées de 65 ans ou plus devrait croître globalement de près de 40 pour cent (croissance annuelle de 2,2 pour cent). Cette croissance globale pourrait être d'au moins 21 pour cent (dans la région de Montréal, où le nombre passerait de 255418 à 309636 individus) et atteindre 83 pour cent (sur la Côte-Nord, où il passerait de 6375 à 11640 individus) voire près de 94 pour cent (Nord-du-Québec, où il passerait de 1101 à 2135 individus) (tableau 6). Ce ne sera donc sans doute ni dans les régions périphériques ni dans les vieux quartiers urbains que les besoins liés à la présence de personnes âgées connaitront la plus grande croissance au cours des années qui viennent, mais bien dans les banlieues des centres urbains plus importants (Mathews, 1988; Mathews, 1996; Bussière et al., 1996).

\section{IMPACTS SUR LA CROISSANCE DE LA POPULATION AVEC INCAPACITÉ}

\section{Évolution de la population avec incapacité, 1991-2006}

Selon les projections, la population avec incapacité crô̂trait, globalement, de plus de 36 pour cent au Québec au cours de la période 1991-2006; pour la population avec incapacité âgée de 65 ans ou plus, l'augmentation devrait avoisiner 45 pour cent (soit une croissance annuelle de 2,5 pour cent) (tableau 7 ). 
TABLEAU 7 - Évolution de la population âgée de 15 ans ou plus et de 65 ans ou plus avec incapacité, selon la catégorie d'incapacité, Québec, 1991-2006

\begin{tabular}{|c|c|c|c|c|c|c|}
\hline \multirow[b]{2}{*}{ INCAPACITÉ } & \multicolumn{3}{|c|}{ Population 15 ans ou plus } & \multicolumn{3}{|c|}{ Population 65 ans ou plus } \\
\hline & 2006 & $\begin{array}{c}\text { Croiss. } \\
\text { globale } \\
1991- \\
2006 \\
(\%)\end{array}$ & $\begin{array}{c}\text { Croiss. } \\
\text { annuelle } \\
1991- \\
2006 \\
(\%)\end{array}$ & 2006 & $\begin{array}{c}\text { Croiss. } \\
\text { globale } \\
1991- \\
2006 \\
(\%)\end{array}$ & $\begin{array}{c}\text { Croiss. } \\
\text { annuelle } \\
1991 \text { - } \\
2006 \\
(\%)\end{array}$ \\
\hline Locom. ambulat. & 540950 & 35,7 & 2,1 & 212371 & 42,6 & 2,4 \\
\hline Locom. faut. roul. & 33890 & 40,6 & 2,3 & 18545 & 50,1 & 2,7 \\
\hline Intellectuelle & 223274 & 37,5 & 2,1 & 103227 & 47,4 & 2,6 \\
\hline Visuelle $^{a}$ & 5193 & 27,4 & 1,6 & 2511 & 54,0 & 2,9 \\
\hline Total & 803307 & 36,3 & 2,1 & 336654 & 44,5 & 2,5 \\
\hline
\end{tabular}

Source : BSQ, ESLA 1986; traitements INRS.

a. Les données sur l'incapacité visuelle doivent être interprétées avec prudence en raison de la faible taille de l'échantillon.

Par catégorie d'incapacité, et abstraction faite de l'incapacité visuelle (données non significatives à cause de la faible taille de l'échantillon), la plus forte croissance globale entre 1991 et 2006 est celle de l'incapacité locomotrice non ambulatoire (fauteuil roulant), pour les personnes âgées de 15 ans ou plus (+ 40 pour cent), et plus encore pour les personnes âgées de 65 ans ou plus (+ 50 pour cent). Même pour l'incapacité locomotrice ambulatoire, la croissance globale attendue pour la période 1991-2006 serait très forte, soit de +36 pour cent chez la population de 15 ans ou plus avec incapacité et de +43 pour cent pour la population âgée de 65 ans ou plus (tableau 7).

\section{Perspectives rêgionales, 1991-2006}

Durant la période 1991-2006, le nombre de personnes avec incapacité âgées de 15 ans ou plus pourrait donc augmenter de plus de 36 pour cent, et celui des personnes âgées de 65 ans ou plus, de près de 45 pour cent. Pour cette même période se confirment les tendances régionales observées en 1991, ainsi que l'importance de la croissance de la population ayant une incapacité pour les régions de Laval, Lanaudière, Laurentides, Montérégie et Outaouais.

La plus forte croissance globale de la population avec incapacité au cours de la période 1991-2006 est celle observée dans les régions urbanisées des Laurentides ( $+64,7$ pour cent), de 
TABLEAU 8 - Évolution de la population âgée de 15 ans ou plus ou de 65 ans ou plus avec incapacité, selon la région, Québec, 19912006

\begin{tabular}{|c|c|c|c|c|c|c|}
\hline \multirow[b]{2}{*}{$\begin{array}{l}\text { RÉGION } \\
\text { ADMINISTRATIVE }\end{array}$} & \multicolumn{3}{|c|}{ Population 15 ans ou plus } & \multicolumn{3}{|c|}{ Population 65 ans ou plus } \\
\hline & 2006 & $\begin{array}{c}\text { Croiss. } \\
\text { globale } \\
1991- \\
2006 \\
(\%)\end{array}$ & $\begin{array}{l}\text { Croiss. } \\
\text { annuelle } \\
1991- \\
2006 \\
(\%)\end{array}$ & 2006 & $\begin{array}{c}\text { Croiss. } \\
\text { globale } \\
1991- \\
2006 \\
(\%)\end{array}$ & $\begin{array}{c}\text { Croiss. } \\
\text { annuelle } \\
1991- \\
2006 \\
(\%)\end{array}$ \\
\hline Bas-Saint-Laurent & 21429 & 20,5 & 1,2 & 9668 & 25,5 & 1,5 \\
\hline Sagu.-Lac-St-Jean & 27157 & 26,8 & 1,6 & 10805 & 49,4 & 2,7 \\
\hline Québec & 79135 & 33,4 & 1,9 & 32683 & 43,7 & 2,4 \\
\hline Mauricie-Bois-Fr. & 53098 & 29,4 & 1,7 & 23464 & 36,4 & 2,1 \\
\hline Estrie & 29838 & 29,5 & 1,7 & 12662 & 29,2 & 1,7 \\
\hline Montréal & 197708 & 20,7 & 1,3 & 99185 & 28,5 & 1,7 \\
\hline Outaouais & 36492 & 55,5 & 3,0 & 12870 & 67,4 & 3,5 \\
\hline Abitibi-Témiscam. & 14073 & 28,4 & 1,7 & 4954 & 31,9 & 1,9 \\
\hline Côte-Nord & 9205 & 33,5 & 1,9 & 3310 & 88,1 & 4,3 \\
\hline Nord-du-Québec & 2438 & 38,1 & 2,2 & 594 & 100,0 & 4,7 \\
\hline Gaspésie-Îles-de-la & M. 10367 & 15,9 & 1,0 & 4547 & 24,7 & 1,5 \\
\hline Chaudière-Appal. & 39183 & 27,9 & 1,9 & 16014 & 36,3 & 2,1 \\
\hline Laval & 35798 & 42,3 & 2,8 & 15551 & 90,4 & 4,4 \\
\hline Lanaudière & 40236 & 52,7 & 3,3 & 13878 & 72,5 & 3,7 \\
\hline Laurentides & 48299 & 64,7 & 3,4 & $17 \quad 142$ & 67,3 & 3,5 \\
\hline Montérégie & 158855 & 53,3 & 2,9 & 59327 & 66,4 & 3.5 \\
\hline Ens. du Québec & 803307 & 36,3 & 2,1 & 336654 & 44,5 & 2,5 \\
\hline
\end{tabular}

Source : BSQ, ESLA 1986; traitements INRS.

l'Outaouais $(+55,5$ pour cent) et de Lanaudière $(+52,7$ pour cent) : ces régions verraient donc plus que doubler leur population à mobilité réduite âgée de 15 ans ou plus (tableau 8). Selon nos prévisions, les taux de croissance seraient encore bien plus élevés pour la population âgée de 65 ans ou plus : par exemple, ils atteindraient $+72,5$ pour cent dans la région de Lanaudière et +67 pour cent dans celle des Laurentides.

Chez la population âgée de 65 ans ou plus ayant une incapacité, l'analyse régionale révèle que le premier rang est occupé par la région métropolitaine de Montréal, suivie de la région de la Montérégie (figures 3 à 5). Les régions de Montréal et de la Montérégie devraient atteindre un niveau similaire en termes de volume de population ayant une incapacité intellectuelle en 2006; la région de Québec occuperait le deuxième rang (figure 6). 


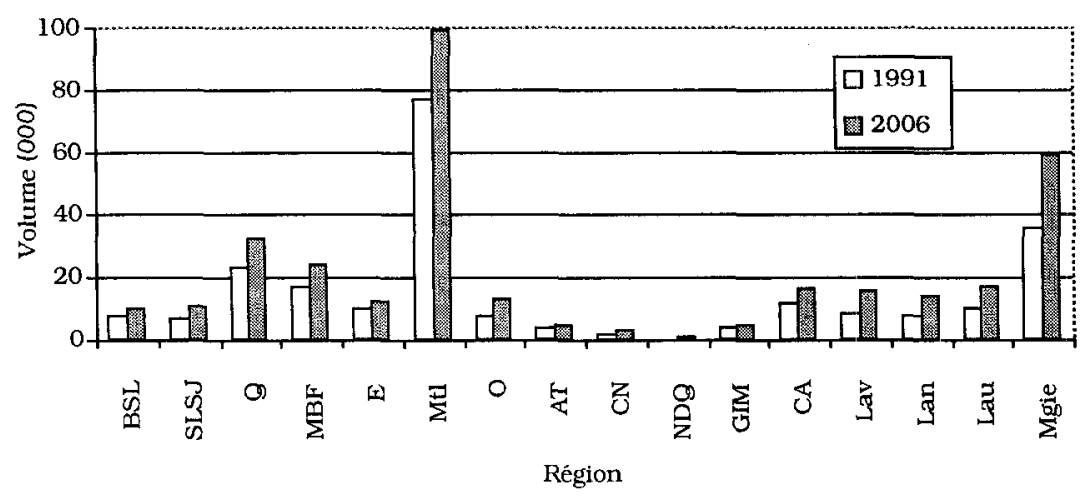

Source : BSQ, ESLA 1986; traitements INRS.

FIGURE 3 -Évolution de la population de 65 ans ou plus ayant une incapacité, par région, Québec, 1991-2006

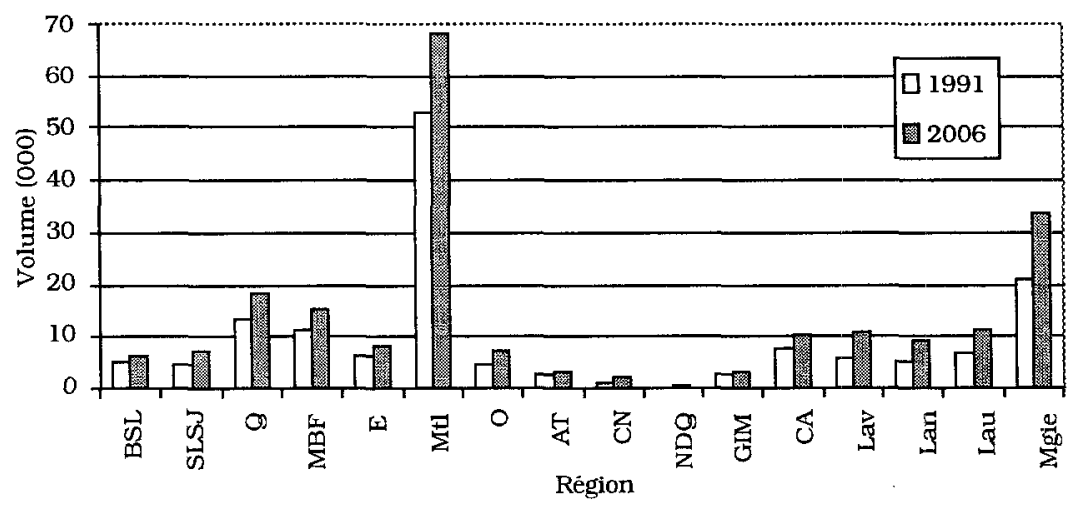

Source : BSQ, ESLA 1986; traitements INRS.

FIGURE 4-Évolution de la population de 65 ans ou plus ayant une incapacité locomotrice ambulatoire, par région, Québec, 1991-2006

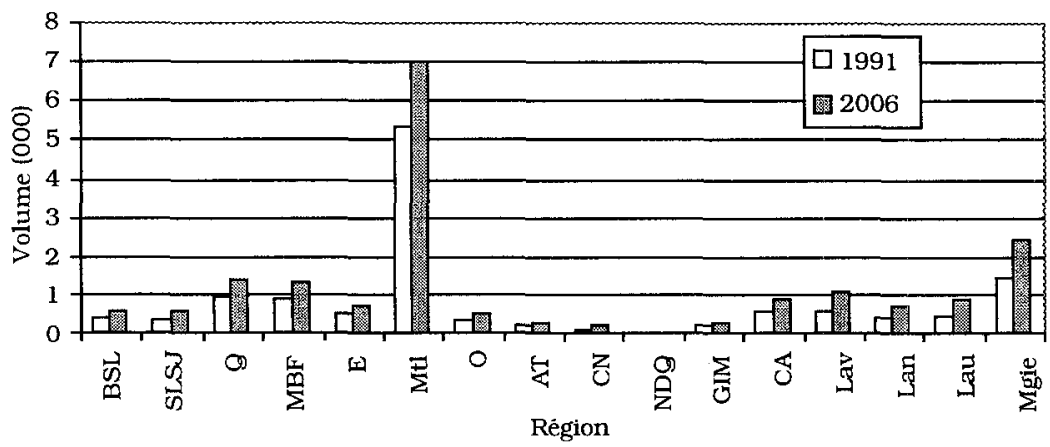

Source : BSQ, ESLA 1986; traitements INRS.

FIGURE 5 -Évolution de la population de 65 ans ou plus ayant une incapacité locomotrice non ambulatoire (fauteuil roulant), par région, Québec, 1991-2006 


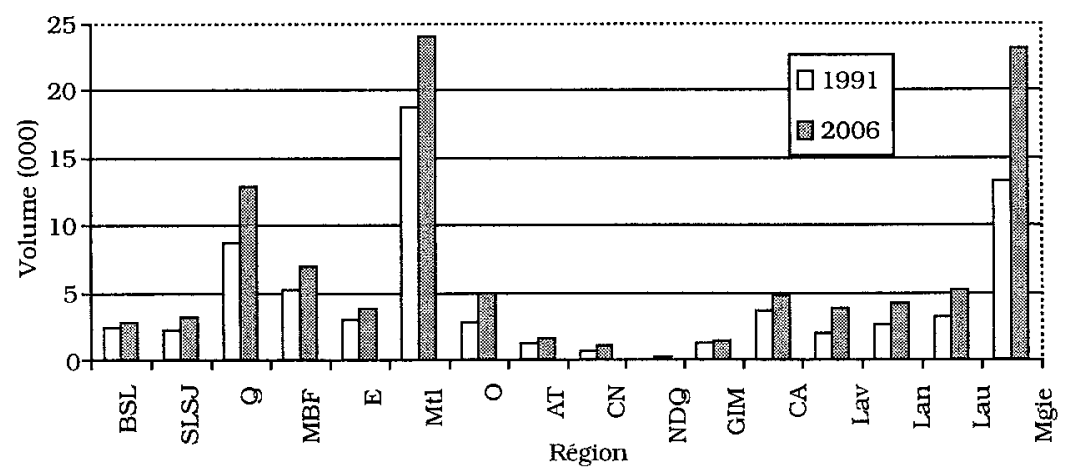

Source : BSQ. ESLA 1986; traitements INRS.

FIGURE 6-Évolution de la population de 65 ans ou plus ayant une incapacité intellectuelle, par région, Québec, 1991-2006

\section{CONCLUSION}

Nous avons projeté l'évolution du nombre de personnes ayant des incapacités qui réduisent leur motricité à l'horizon 1991-2006 pour le ministère des Transports du Québec (MTQ). Nous étions partagés entre les besoins opérationnels d'un ministère pour lequel un horizon de 15 ans est très long, et la curiosité qui pousse le chercheur à extrapoler des tendances sur un horizon encore plus lointain, sachant, d'une part, que l'incapacité augmente sensiblement avec l'âge de l'individu (Thouez et al., 1993) et, d'autre part, que le phénomène du vieillissement de la population au Guébec atteindra sa phase critique autour de l'année 2011. Le choix de l'horizon 19912016 aurait assuré des résultats spectaculaires.

Cependant, même sur un horizon jugé relativement court en démographie (15 ans), et avec des données régionales plutôt grossières au niveau des incapacités, les variations démographiques amènent à elles seules des écarts régionaux importants en ce qui concerne l'évolution de la perte de mobilité des populations. Il n'est cependant pas évident qu'à la croissance du volume des clientèles potentielles de certains services spécialisés correspondra une augmentation proportionnelle du niveau des besoins réels exprimés, car d'autres facteurs interviennent, à savoir les comportements des usagers. Nous avons volontairement omis ici cette question, traitée ailleurs (voir en particulier Bussière, 1990; Bussière, Thouez et Laroche, 1993; Bussière et Bernard, 1995; Bussière et al., 1996; Bussière, Bernard et Thouez, 1997), pour centrer l'analyse sur les dimensions purement démographiques du phénomène. 


\section{RÉFÉRENCES BIBLIOGRAPHIUUES}

BUSSIĖRE, Yves, 1990. "Vieillissement spatialisé, demande de transport et prospective : le cas montréalais 1986-2011", Cahiers québécois de démographie, 19, $2: 325-350$.

BUSSIĖE, Yves, Anne BERNARD et Jean-Pierre THOUEZ, 1997. Vieillissement et demande de transport adapté au Québec: perspectives régionales, 1993-2006. Texte préparé pour présentation au colloque scientifique international "Ville et vieillissement", Arles, France, 8-10 octobre 1997, 17 p. À paraitre dans les actes du colloque.

BUSSIÈRE, Yves, Jean-Pierre THOUEZ et Pierre LAROCHE, 1993. "Vieillissement et demande de transport des personnes à mobilité réduite : un modèle de prospective appliqué au cas montréalais : 1986-2011", Routes et transport, Québec, 23, 3 : 34-44.

BUSSIERE, Yves, Jean-Pierre THOUEZ, Ron G. RICE, Georges MATHEWS et Anne BERNARD, 1996. Portrait et prévisions de la clientèle à mobilité réduite en transport au Québec, 1993-2006. 3 tomes, 65, 309 et 270 p. Montréal, INRS-Urbanisation et ministère des Transports du Québec.

BUSSIÈRE, Yves, et Anne BERNARD, 1995. Femmes âgées, motorisation et autonomie dans la région métropolitaine de Montréal: perspectives d'avenir centre-périphérie. Communication présentée au 19e colloque annuel de l'ACSR, UGAM, 3-5 juin. Montréal, INRS-Urbanisation, $27 \mathrm{p}$.

CANADA, Statistique Canada, 1991. Enquêtes ESLA 1986 et 1991 let guide de l'utilisateur ESLA 1991]. Ottawa, Ontario.

COLVEZ, Alain, Denis BUCgUET, Sarah CURTIS et Robert PAMPALON, 1990. Approche épidémiologique des besoins en services pour les personnes âgées dépendantes, Groupes cibles : Incap. : Aides professionnelles et non professionnelles, Montpellier, INSERM, $45 \mathrm{p}$.

GUYON, Louise, et M. LEVASSEUR, 1991. Les Variables: catégorisation, indicateurs et indices de Santé Québec, Cahier technique 87-08. Québec, Ministère de la Santé et des Services sociaux et DSC.

MATHEWS, Georges, 1988. Le Vieillissement démographique et son impact sur la situation des personnes âgées et les services qui leur sont offerts. Québec, Conseil québécois de la recherche sociale, $136 \mathrm{p}$.

MATHEWS, Georges, 1996. "L'avenir démographique des régions", Recherches sociographiques, XXXVII, 3.

QUÉBEC, Province, 1993. Politique d'admissibilité au transport adapté. Québec, ministère des Transports. 
QUÉBEC, Province, 1994a. Répertoire statistique du transport adapté 1993. Ministère des Transports, Direction génêrale transport terrestre des personnes.

GUÉBEC, Province, 1994b. Une nouvelle politique d'admissibilité au transport adapté entre en vigueur dès le ler janvier 1994, le saviez-vous ? Ministère des Transports du Québec, $12 \mathrm{p}$.

QUÉBEC, Province, Bureau de la statistique du Québec, 1995. Perspectives démographiques du Québec et de ses régions : 19912041 et des MRC: 1991-2016. Les Publications du Québec.

SAUCIER, Alain, 1992. Le Portrait des personnes ayant des incapacités au Québec en 1986. Ministère de la Santé et des Services sociaux, Direction de l'évaluation, collection "Données, statistiques et indicateurs".

SAUCIER, Alain, et P. LAFONTAINE, 1991. Nature et sévérité des incapacités au Québec en 1986. Un portrait statistique des adultes à domicile et en établissement selon l'enquête ESLA, Ministère de la Santé et des Services sociaux, $47 \mathrm{p}$.

THOUEZ, Jean-Pierre, Yves BUSSIEERE, Robert PAMPALON et Nathalie CHICOINE, 1993. "Vieillissement et limitations fonctionnelles : analyse comparée des données de l'enquête ESLA entre la région métropolitaine de Montréal et la province de Québec, 1986", Cahiers québécois de démographie, 22, 1 : 45-62.

THOUEZ, Jean-Pierre, Yves BUSSIERE, Anne BERNARD et Pierre LAROCHE, 1996. Prévisions régionales des clientèles âgées à mobilité réduite au Québec et indicateurs de besoins. Texte préparé pour présentation au colloque de l'Association internationale des démographes de langue française, Sinaia, Roumanie, septembre 1996, 12 p. À paraitre dans les actes du colloque. 
BERNARD Anne, BUSSIĖRE Yues et THOUEZ Jean-Pierre - VIEILLSSEMENT ET INCAPACITÉS AU QUÉBEC : PERSPECTIVES RÉGIONALES 1991-2006

Le vieillissement rapide de la population québécoise au cours des prochaines décennies entraînera des disparités régionales importantes. À partir de projections démographiques régionales du Bureau de la statistique du Québec (BSQ) sur la période 1991-2006 et des données de l'Enquête sur la santé et les limitations d'activités de Statistique Canada (ESLA), nous présentons une analyse de l'évolution des clientèles à mobilité réduite. L'analyse démontre non seulement l'importance des disparités régionales mais également leur impact sur l'évolution des incapacités restreignant la mobilité.

BERNARD Anne, BUSSIÈRE YUes and THOUEZ Jean-Pierre-AGING AND DISABILITY IN QUEBEC: REGIONAL PROJECTIONS 1991-2006

The rapid aging of Quebec's population in the next few decades will entail significant regional disparities. Based on regional demographic projections by the Bureau de la statistique du Québec (BSQ) for the years 1991-2006 and data from Statistics Canada's Health and Activity Limitation Survey (HALS), we analyze changes in clienteles with mobility impairments. This analysis shows not only the extent of regional disparities but also their impact on the evolution of disabilities that restrict mobility.

BERNARD Anne, BUSSIĖRE Yues y THOUEZ Jean-PieTre - ENVEJECIMIENTO E INCAPACIDAD EN QUEBEC : PERSPECTIVAS REGIONALES 1991-2006

El rápido envejecimiento de la población quebequense en el curso de las próximas décadas provocará disparidades regionales importantes. A partir de proyecciones demográficas regionales de la oficina de estadística de Quebec (Bureau de la statistique du Québec, BSQ) sobre el periodo 1991-2006, y de datos de la Encuesta sobre la Salud y las Limitaciones de Actividades de Statistique Canada (ESLA), se presenta aquí un análisis de la evolución de las clientelas con movilidad reducida. El análisis demuestra no sólo la importancia de las disparidades regionales, sino también su impacto en la evolución de las incapacidades que restringen la movilidad. 